\title{
Aplicação aérea de fungicidas na cultura do arroz irrigado com diferentes bicos de pulverização
}

\author{
Aerial fungicide application on irrigated lowland rice with varying spraying nozzles
}

\section{Tânia Bayer ${ }^{\mathrm{I}}$ Adriano ArruéII Ivan Francisco Dressler da Costa $^{\mathrm{III}}$ Giuvan Lenz ${ }^{\mathrm{III}}$ Cezar Coradini ${ }^{\mathrm{II}}$ Bruno Giacomini Sari ${ }^{\mathrm{III}}$ Maiquel Pizzuti Pes ${ }^{\mathrm{II}}$}

\section{RESUMO}

Na safra agrícola 2007/2008, foi realizado um estudo com o objetivo de avaliar a deposição e penetração de gotas através de cartões hidrossensíveis e análise cromatográfica. Para isso, utilizaram-se o bico eletrostático, bico hidráulico e atomizadores rotativos de discos com diferentes volumes de calda. As pulverizações foram realizadas no estádio $R 3$ da cultura, com aeronave Ipanema EMB-202, aplicandose fungicida trifloxistrobina + propiconazole. Através da leitura de cartões hidrossensíveis, determinou-se a densidade de gotas, o diâmetro médio numérico, diâmetro médio volumétrico, amplitude relativa e a penetração de gotas no dossel (\%). Também foi utilizada a análise cromatográfica para determinar a penetração do produto no alvo biológico. O bico hidráulico,

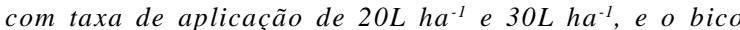

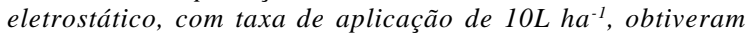
maior densidade de gotas no terço superior. $O$ uso de atomizadores rotativos de disco com volume de calda de $15 \mathrm{~L}$ ha ${ }^{-1}$ apresentou maior densidade de gotas no terço médio e inferior. Para diâmetro médio volumétrico, os bicos eletrostáticos juntamente com os atomizadores rotativos de discos apresentaram os menores valores. A maior homogeneidade de gotas no terço superior foi obtida com atomizadores rotativos de disco com taxa de aplicação de $6 L$ $h a^{-1}$ e $10 L h^{-1}$. A análise cromatográfica mostrou que a maior quantidade de produto foi retida com bicos eletrostáticos com taxa de aplicação de $10 \mathrm{~L} \mathrm{ha} \mathrm{L}^{-1}$ no extrato inferior da planta.

Palavras-chave: bico hidráulico, bico eletrostático e atomizador rotativo de disco.

\section{ABSTRACT}

In the harvest year of 2007/2008 a study was carried to evaluate the droplets deposition and penetration throughout water sensible cards and gas chromatographic analysis. Were used the hydraulic nozzle, electrostatic nozzle and rotary-disk atomizer spraying systems, with different volumes of spray solution. Aerial applications were performed at R3 stage using the aircraft Ipanema EMB-202 and the fungicide trifloxystrobin + propiconazole. Throughout the lection of the water sensible cards was determinate the droplet density, the medium numeric diameter, the medium volumetric diameter, the relative amplitude and the percentage of droplet penetration in the canopy. Chromatographic analysis was also performed to determinate the fungicide penetration into the biological target.

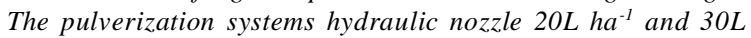

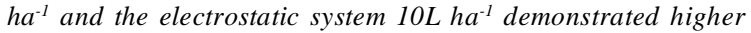
droplet density in the upper third. The rotary-disk atomizer $15 \mathrm{~L}$ ha $a^{-1}$ showed higher droplet density in the mid and low third. To the medium volumetric diameter, the electrostatic nozzles and the rotary-disk atomizer showed the lowest values. The higher homogeneity in the upper third was attained with rotary-disk atomizer at the pulverization rates of $6 \mathrm{~L} \mathrm{ha} \mathrm{I}^{-1}$ and $10 \mathrm{~L} \mathrm{ha} \mathrm{C}^{-1}$. The chromatographic analysis showed that the higher quantity of product retention was obtained with the electrostatic system at the pulverization rate of $10 \mathrm{~L} \mathrm{ha} \mathrm{L}^{-1}$ in the lower plant extract.

Key words: hydraulic nozzle, electrostatic nozzle and rotarydisk atomizer.

\section{INTRODUÇÃO}

A cultura do arroz destaca-se no setor agrícola mundial por sua importância socioeconômica, uma vez que é base da alimentação humana em diversos países. Dados da FAO (2009) mostram que cerca de

'Departament d'Enginyeria Agroalimentària i Biotecnologia, Universitat Politècnica de Catalunya (UPC), Castelldefels, Catalunha, Espanha.

"Departamento de Defesa Fitossanitária, Universidade Federal de Santa Maria (UFSM), Av. Roraima, 1000, Cidade Universitária, 97105-900, Santa Maria, RS, Brasil. E-mail: adrianoarrue@ hotmail.com. Autor para correspondência.

IIIDepartamento de Defesa Fitossanitária, UFSM, Santa Maria, RS, Brasil. 
$86 \%$ do arroz produzido no mundo é utilizado para a alimentação humana. No Brasil, o arroz ocupa posição de destaque do ponto de vista econômico, com uma área de 2,45 milhões de hectares, produzindo 13,61 milhões de toneladas de grãos na safra 2011/2012 (CONAB, 2012). Atualmente, há necessidade de aumentar a produtividade dessa cultura, com o objetivo de atender à crescente demanda decorrente do aumento da população mundial. Para que esse aumento ocorra, são necessárias práticas que diminuam perdas e, dentre estas, destaca-se o controle de doenças através da aplicação de fungicidas em parte aérea, comumente utilizada em lavouras do estado do Rio Grande do Sul.

As doenças fúngicas no arroz irrigado são responsáveis por perdas na produtividade de até $50 \%$ (BALARDIN \& BORIN, 2001), sendo que o seu controle está relacionado com a eficiência dos fungicidas. Essa eficiência é influenciada, entre outros fatores, pela correta distribuição e uniformidade do produto no alvo (MENEGHETTI, 2006). Nesse sentido, ao se dar muita importância ao produto e pouca atenção a tecnologia de aplicação, tem-se como consequência a diminuição da eficiência da aplicação (CUNHA et al., 2003; CUNHA \& RUAS, 2006).

Entre os fatores que influenciam na qualidade de aplicação, destacam-se a seleção e regulagem dos equipamentos, a aplicação em condições climáticas adequadas e a deposição na superfície a ser tratada (BALAN et al., 2006). Assim sendo, o conhecimento da tecnologia de aplicação é fundamental para a eficácia da pulverização. Além disso, a definição do volume de calda é outro fator importante a ser observado. $\mathrm{O}$ volume a ser aplicado vai depender do tipo de alvo, do tamanho das gotas, da cobertura necessária, da forma de ação do defensivo e da tecnologia de aplicação, dentre outros fatores (ANTUNIASSI \& BAIO, 2008).

A utilização de novas tecnologias de aplicação leva a questionamentos sobre a capacidade de penetração da calda no dossel das plantas (CUNHA et al., 2011). Dentre essas tecnologias, destacam-se as aplicações aéreas, que, segundo REZENDE (2011), têm sido amplamente utilizadas no Brasil, principalmente devido à sua maior capacidade operacional em relação à aplicação terrestre. Por outro lado, estas novas tecnologias levam a questionamentos quanto à taxa de aplicação recomendada, sendo necessários estudos para a determinação do melhor volume a ser aplicado (CUNHA et al., 2011).

As técnicas e equipamentos de pulverização são avaliados por estudos de deposição das gotas no dossel. O uso de cartões hidrossensíveis é recomendado por CARVALHO (1995) para avaliar as pulverizações aéreas e terrestres quanto à densidade e o diâmetro médio volumétrico de gotas, assim como a penetração da calda no dossel. Outra possibilidade é a utilização de marcadores específicos, ou o próprio ingrediente ativo do produto utilizado, que, sendo detectado nas partes medianas e inferiores do dossel, constatariam a eficiência da aplicação, devido à baixa mobilidade do produto na planta (CUNHA, et al., 2011; REZENDE, 2011).

Nesse contexto, o trabalho teve como objetivo avaliar a deposição e a penetração de gotas no dossel da cultura do arroz irrigado, gerados por bicos hidráulicos, eletrostáticos e atomizadores rotativos de discos, com diferentes volumes de calda, por meio de cartões hidrossensíveis e da análise cromatográfica.

\section{MATERIAL E MÉTODOS}

O experimento foi conduzido no município de Rio Grande - RS, na safra agrícola 2007/2008, em lavoura comercial com a cultivar 'Qualimax 1', densidade de semeadura de $85 \mathrm{~kg} \mathrm{ha}^{-1}$ e espaçamento de $0,17 \mathrm{~m}$ entre linhas. Os tratos culturais foram realizados conforme as recomendações técnicas para a cultura do arroz (SOSBAI, 2005). Os tratamentos constaram de pulverizações com diferentes bicos e volumes, realizados com auxílio de uma aeronave. Os bicos de pulverização e os diferentes volumes de calda utilizados foram: bico hidráulico e volume de calda de $30 \mathrm{~L} \mathrm{ha}^{-1}$ (BH 30); bico hidráulico e volume de calda de 20 $\mathrm{L} \mathrm{ha}^{-1}$ (BH 20); bico eletrostático e volume de calda de 10L ha ${ }^{-1}$ (BE 10); bico eletrostático e volume de calda de $5 \mathrm{~L} \mathrm{ha}^{-1}$ (BE 5); atomizador rotativo de disco e volume de calda de $15 \mathrm{~L}$ $\mathrm{ha}^{-1}$ (ARD 15); atomizador rotativo de disco e volume de calda de 10 $\mathrm{L} \mathrm{ha}^{-1}$ (ARD 10); atomizador rotativo de disco e volume de calda de 6 $\mathrm{L} \mathrm{ha}^{-1}$ (ARD 6).

Cada tratamento foi aplicado em talhões medindo 400x225 metros, perfazendo 15 faixas de vôo da aeronave com 15 metros de largura cada, em uma área total de nove hectares por tratamento. Em cada talhão foram alocadas sete áreas de avaliações medindo 10x6 metros, representando o número de repetições. As áreas foram georreferenciadas e demarcadas com estacas.

A aeronave utilizada foi Ipanema modelo 202, com equipamentos previamente montados em barras distintas. O fungicida utilizado constou da formulação comercial de dois ingredientes ativos: Trifloxistrobina + Propiconazol, na dose de $0,75 \mathrm{~L} \mathrm{ha}^{-1}$, com concentração de ingrediente ativo de $125 \mathrm{~g}+125 \mathrm{~g}$, respectivamente, sendo aplicado no estádio R3 da cultura (COUNCE et al., 2000). As condições micrometeorológicas no momento das aplicações eram: temperatura de $24,6^{\circ} \mathrm{C} \pm 0,5$, umidade relativa do ar $70,6 \% \pm 3,7$ e $5,8 \mathrm{~m} \mathrm{~s}^{-1} \pm 1,9$ de velocidade média do vento. 
$\mathrm{Na}$ avaliação de deposição e penetração de gotas no dossel, foram utilizadas estacas com um metro de comprimento, nas quais foram alocados cartões hidrossensíveis em posição horizontal em relação à cultura a 0,3, 0,6 e 0,9 metros do solo. Esses cartões foram coletados logo após a pulverização, envoltos em papel alumínio de forma individual para evitar contato com a umidade e enviados para análise. A metodologia utilizada partiu da captura de imagem dos cartões com scanner em área de, no mínimo, um centímetro quadrado, com posterior análise da imagem digitalizada através do software Agroscan.

Os parâmetros analisados foram o diâmetro médio numérico (DMN), diâmetro médio volumétrico (DMV) e a densidade de gotas. A penetração (\%) foi calculada com base na densidade de gotas no terço superior da cultura, de modo que este representou $100 \%$. Assim sendo, a penetração de gotas é a relação entre a densidade de gotas do terço médio e inferior, comparado ao terço superior. Além disso, com base nos dados de DMV e DMN, foi calculada a amplitude relativa (AR), considerada parâmetro mais consistente para avaliação da homogeneidade de gotas (OZEKI, 2006).

Para a realização da análise cromatográfica, as plantas foram colhidas de forma aleatória dentro dos talhões, pesados 400 gramas de folhas e colmo e divididas ao meio (parte superior e inferior). Essas amostras foram embaladas em papel alumínio, acondicionadas em sacos plásticos e levadas para análise no Laboratório de Análise de Resíduos de Pesticidas (LARP). O marcador utilizado foi o ingrediente ativo Trifloxistrobina, presente no produto comercial.

O preparo das amostras foi efetuado pelo método de extração QuEChERS modificado (ANASTASSIADES et al., 2003). Inicialmente, foram pesados $\pm 10 \mathrm{~g}$ de amostra, previamente homogeneizada, diretamente em tubos de polipropileno de $50 \mathrm{~mL}$ com tampa. Adicionaram-se $10 \mathrm{~mL}$ de acetonitrila contendo $1 \%$ de ácido acético, em cada tubo, e, após fechá-los, efetuou-se agitação manual e vigorosa por cerca de 15 segundos. Em seguida, acrescentaram-se 3,0 e 1,7g de sulfato de magnésio anidro e acetato de sódio anidro, respectivamente. Repetiram-se as agitações manuais por um minuto. Os tubos tampados foram centrifugados a 4000rpm, durante oito minutos, e, posteriormente, transferiram-se diretamente $4 \mathrm{~mL}$ do extrato líquido para outro tubo de polipropileno de $15 \mathrm{~mL}$, já contendo outros 500mg de sulfato de magnésio anidro, $100 \mathrm{mg}$ de adsorvente amina primária secundária (PSA) e 100mg de carvão ativado, sendo novamente agitados vigorosa e manualmente, por cerca de 20 segundos, e novamente centrifugado. A solução resultante foi analisada por Cromatografia Gasosa acoplada à Espectrometria de
Massas (GC-MS), no modo de ionização química negativa e modo de aquisição por monitoramento do íon selecionado.

Os valores obtidos foram submetidos à análise de variância. Os dados de densidade e penetração de gotas, diâmetro médio volumétrico e amplitude relativa foram submetidos à transformação Box - Cox. Após a verificação da normalidade dos erros e das variâncias, utilizou-se o teste de comparação de médias de Skott - Knott, em nível de probabilidade de erro de 5\%, com auxílio do software Assistat ${ }^{\circledR}$.

\section{RESULTADOS E DISCUSSÃO}

Os resultados de densidade de gotas no terço superior indicaram diferenças entre os equipamentos $\mathrm{e}$ os volumes testados. Os tratamentos BH 30, BH 20 e BE 10 não diferiram estatisticamente entre si, em relação à densidade de gotas, apresentando valores entre 96 e 139 gotas $\mathrm{cm}^{-2}$ (Figura 1). A densidade de gotas encontrada nos demais tratamentos foi sempre superior a 40 gotas $\mathrm{cm}^{-2}$, mesmo quando se testaram equipamentos com baixo volume de calda, como no caso do tratamento BE 5. A cobertura doalvo está relacionada com a eficiência da aplicação, sendo que, de acordo com OZEKI (2006), uma faixa de densidade de gotas de 30 a 40 gotas $\mathrm{cm}^{-2}$ seria ideal para fungicidas sistêmicos.

Com relação ao terço médio, a densidade de gotas diferiu quando se utilizaram volumes de calda superiores a $15 \mathrm{~L} \mathrm{ha}^{-1}$, independente do bico utilizado. Os tratamentos BH 30, BH 20 e ARD 15 apresentaram as maiores densidades, com valores entre 20 e 30 gotas $\mathrm{cm}^{-2}$, sendo que os demais apresentaram valores inferiores a 20 gotas $\mathrm{cm}^{-2}$.

No terço inferior, os tratamentos ARD $15 \mathrm{e}$ BH 20 apresentaram as maiores densidade de gotas, com densidades de 25 gotas $\mathrm{cm}^{-2}$ e 14 gotas $\mathrm{cm}^{-2}$, respectivamente. Destaca-se novamente a baixa densidade de gotas dos tratamentos com baixo volume de calda (BE 10, BE 5, ARD 10 e ARD 6), que apresentaram densidades abaixo de 10 gotas $\mathrm{cm}^{-2}$.

Além da cobertura do alvo, a capacidade de penetração das gotas vai afetar a eficiência biológica da aplicação (OZEKI \& KUNZ, 1998). A análise da penetração de gotas no dossel mostrou que o tratamento BH 30 apresentou uma baixa capacidade de penetração, levando a menores densidades de gota no terço inferior, mesmo tendo obtido a maior densidade de gotas no terço superior. Já em relação ao tratamento ARD 15, observa-se uma grande capacidade de penetração, levando a uma maior densidade no terço inferior, em virtude do atomizador rotativo de discos produzir gotas com diâmetro reduzido. Apesar de não diferirem do 


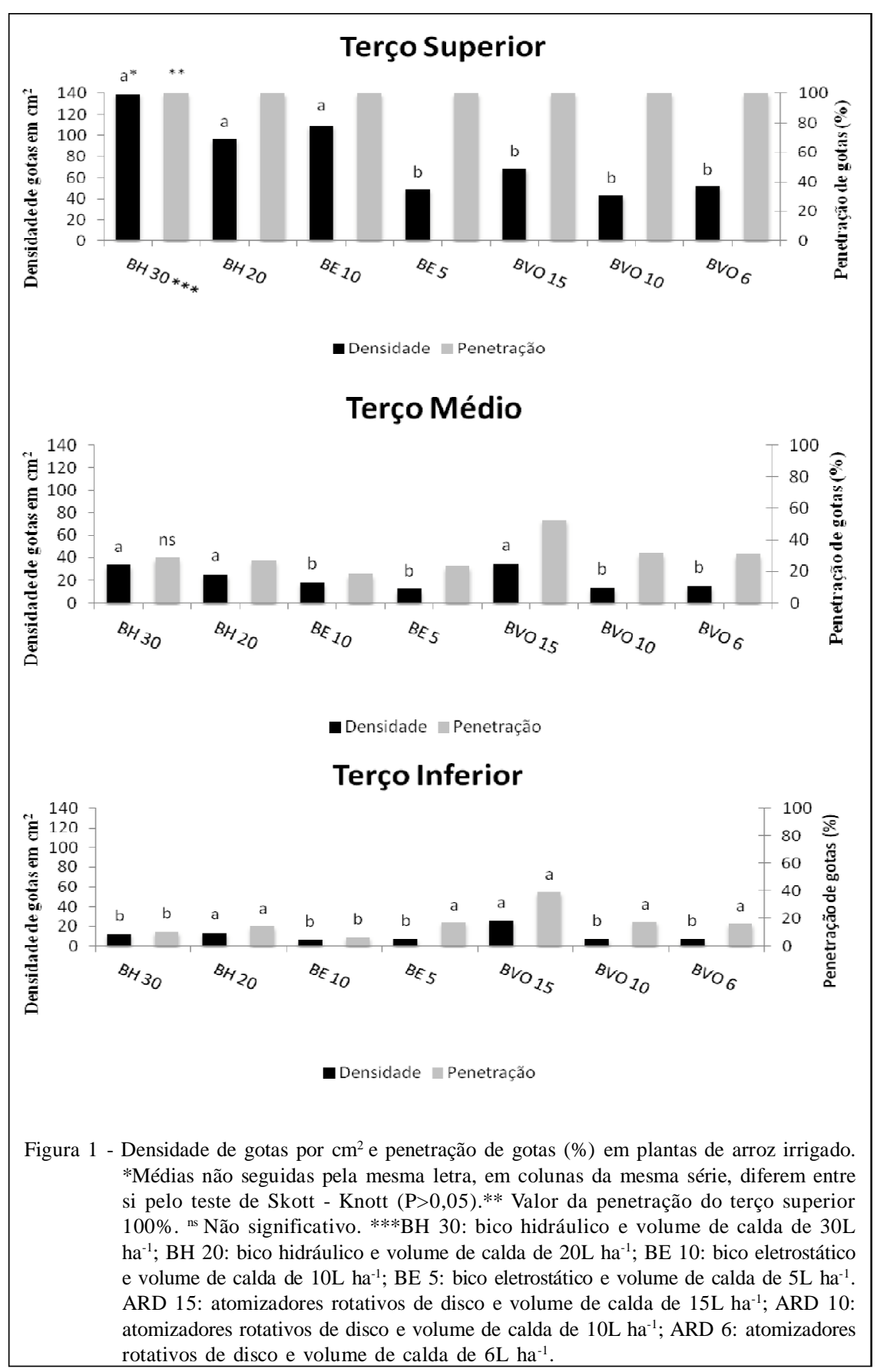

tratamento ARD 15 quanto à penetração de gotas, os tratamentos BE 5, BE 10 e ARD 6 apresentaram baixas densidades de gota no terço inferior.

Os resultados ratificaram a relação entre o aumento do volume de calda e a densidade de gotas, que já havia sido encontrado por SCHRÖDER (1996), quando observou que aplicações com maiores volumes de calda apresentaram maior densidade de gotas. Cabe ressaltar que apenas no tratamento $\mathrm{BH} 20$, no terço inferior, esse fato não ocorreu, pois o tratamento apresentou uma densidade de gotas superior ao $\mathrm{BH}$ 30. O resultado inverso, observado nesse caso, pode estar relacionado à dificuldade dos cartões hidrossensíveis de detectar gotas mais finas pulverizadas pelo tratamento $\mathrm{BH} 30$, uma vez que este apresentou um DMN menor que o primeiro. 
Em relação aos tratamentos BE 10, BE 5 e ARD 6, é possível observar o baixo DMV de gota gerado, que apresenta grande potencial de deriva (Tabela 1). Quanto maior o DMV das gotas geradas pelos bicos de pulverização, menores são os percentuais de gotas com tamanho inferior que 100 $\mu \mathrm{m}$ (CUNHA et al., 2011). O problema de deriva é potencializado por gotas com DMV menores que $100 \mu \mathrm{m}$, sendo que, para uma aplicação segura, gotas desse diâmetro não devem representar mais de $15 \%$ do volume pulverizado (CUNHA et al., 2003). Os baixos valores de DMN também confirmam tal afirmação, uma vez que, por dividir o diâmetro de gotas de forma aritmética, esse parâmetro mostra que grande quantidade de gotas finas foram geradas. Segundo MOTA(2011), o valor de DMN é influenciado pelas gotas menores (quantidade).

Nota-se que os equipamentos com bico hidráulico apresentaram as maiores variações no tamanho de gotas, juntamente com o tratamento BE 10. No caso do tratamento $\mathrm{BH} 30$, foi verificado um conjunto de gotas heterogêneo, que, segundo MATUO et al. (2001), ocorre quando os valores de amplitude relativa são maiores que 1,4 , indicando a produção de gotas grossas. De acordo ANTUNIASSI \& BAIO (2008), as gotas finas estão relacionadas com a penetração no dossel das plantas, uma vez que a cobertura proporcionada é maior do que com gotas mais grossas, o que pode explicar o fato de esse tratamento ter mostrado uma baixa capacidade de penetração.

Além da quantificação de parâmetros relacionados à eficiência da aplicação através do uso de cartões hidrosensíveis, buscou-se determinar a deposição do produto no estrato superior e inferior da cultura através da utilização da análise cromatográfica. Em relação ao estrato superior, não houve diferença estatística entre os tratamentos. Porém, em relação ao estrato inferior (Figura 2), os resultados apontam maior quantidade de produto depositado no tratamento $\mathrm{BE}$ $10 \mathrm{~L} \mathrm{ha}^{-1}$, não diferindo do $\mathrm{ARD} 15 \mathrm{~L} \mathrm{ha}^{-1}$ e $6 \mathrm{~L} \mathrm{ha}^{-1}$. Diferentemente dos resultados encontrados com a análise dos papéis hidrossensíveis, não se observou baixa capacidade de penetração e cobertura nos tratamentos ARD 6 $\mathrm{La}^{-1}$ e BE $10 \mathrm{~L} \mathrm{ha}^{-1}$, demonstrando que a utilização de papéis hidrossensíveis não foi adequada para quantificar gotas menores geradas por esses bicos de pulverização.

Apesar de ser recomendado para avaliar pulverizações aéreas e terrestres, o uso de papéis hidrossensíveis pode apresentar limitações, principalmente quando se avaliam equipamentos que geram gotas muito pequenas ou com indução de carga. SCHRÖDER (2003) destaca que esse tipo de análise subestima os resultados, seja pela sensibilidade do scanner ou pela ausência de carga no papel.

\section{CONCLUSÃO}

Nas condições em que o trabalho foi desenvolvido, conclui-se que taxas de aplicação acima de $15 \mathrm{~L} \mathrm{ha}^{-1}$ proporcionaram maior densidade de gotas no estrato superior e médio do dossel foliar. Além disso, equipamentos que geram gotas menores propiciam maior penetração delas no terço inferior do dossel.

A análise cromatográfica mostrou-se o melhor método de identificação de penetração de calda no estrato inferior de plantas de arroz. Os papéis

Tabela 1 - Diâmetro Médio Numérico (DMN), Diâmetro Médio Volumétrico (DMV) e Amplitude Relativa (AR) de gotas nos terços superior, médio e inferior da cultura.

\begin{tabular}{|c|c|c|c|c|c|c|c|c|c|}
\hline & \multicolumn{3}{|c|}{ 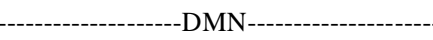 } & \multicolumn{3}{|c|}{ - } & \multicolumn{3}{|c|}{ 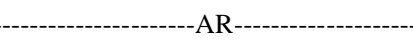 } \\
\hline & Superior & Médio & Inferior & Superior & Médio & Inferior & Superior & Médio & Inferior \\
\hline $\mathrm{BH} 30 * *$ & $107,8 \mathrm{c}^{*}$ & $98,8 \mathrm{~b}$ & $106,7 \mathrm{a}$ & $261,6 \mathrm{a}$ & $156,3^{\text {ns }}$ & $177,0 \mathrm{a}$ & $1,47 \mathrm{~b}$ & $1,22 \mathrm{a}$ & $1,04^{\mathrm{ns}}$ \\
\hline BH 20 & $117,5 \mathrm{~b}$ & $110,5 \mathrm{a}$ & $102,6 \mathrm{a}$ & $245,6 \mathrm{a}$ & 154,6 & $221,1 \mathrm{a}$ & $1,32 \mathrm{~b}$ & $0,88 \mathrm{~b}$ & 1,11 \\
\hline BE 10 & $90,7 \mathrm{~d}$ & $85,6 \mathrm{~b}$ & $80,3 \mathrm{~b}$ & $159,8 \mathrm{c}$ & 137,0 & $131,1 \mathrm{~b}$ & $1,38 \mathrm{~b}$ & $1,06 \mathrm{a}$ & 0,98 \\
\hline BE 5 & $89,8 \mathrm{~d}$ & $88,5 \mathrm{~b}$ & $80,1 \mathrm{~b}$ & $129,3 \mathrm{~d}$ & 131,1 & $134,8 \mathrm{~b}$ & $1,06 \mathrm{a}$ & $1,01 \mathrm{a}$ & 1,01 \\
\hline ARD 15 & $131,0 \mathrm{a}$ & $119,6 \mathrm{a}$ & $109,0 \mathrm{a}$ & $209,0 \mathrm{~b}$ & 153,1 & $148,3 \mathrm{~b}$ & $1,15 \mathrm{a}$ & $1,17 \mathrm{a}$ & 1,09 \\
\hline ARD 10 & $121,3 \mathrm{~b}$ & $111,0 \mathrm{a}$ & $99,7 \mathrm{a}$ & $175,6 \mathrm{c}$ & 137,5 & $139,1 \mathrm{~b}$ & $1,03 \mathrm{a}$ & $0,73 \mathrm{~b}$ & 0,88 \\
\hline ARD 6 & $103,5 \mathrm{c}$ & $102,8 \mathrm{a}$ & $104,5 \mathrm{a}$ & $144,3 \mathrm{~d}$ & 122,1 & $135,5 \mathrm{~b}$ & $1,03 \mathrm{a}$ & $0,73 \mathrm{~b}$ & 0,89 \\
\hline $\mathrm{CV}(\%)$ & 7,8 & 10,4 & 19,4 & 13,4 & 9,9 & 19,9 & 6,9 & 18,9 & 31,1 \\
\hline
\end{tabular}

*Médias não seguidas pela mesma letra, na coluna, diferem entre si pelo teste de Skott - Knott $(\mathrm{P}>0,05)$. **BH 30: bico hidráulico e volume de calda de 30L ha ${ }^{-1}$; BH 20: bico hidráulico e volume de calda de 20L ha ${ }^{-1}$; BE 10: bico eletrostático e volume de calda de $10 \mathrm{~L}$ ha ${ }^{-1}$; BE 5: bico eletrostático e volume de calda de $5 \mathrm{~L} \mathrm{ha}^{-1}$; ARD 15: atomizadores rotativos de disco e volume de calda de $15 \mathrm{~L}$ ha ${ }^{-1}$; ARD 10 : atomizadores rotativos de disco e volume de calda de 10L ha ${ }^{-1}$; ARD 6: atomizadores rotativos de disco e volume de calda de 6L ha ${ }^{-1}$. 


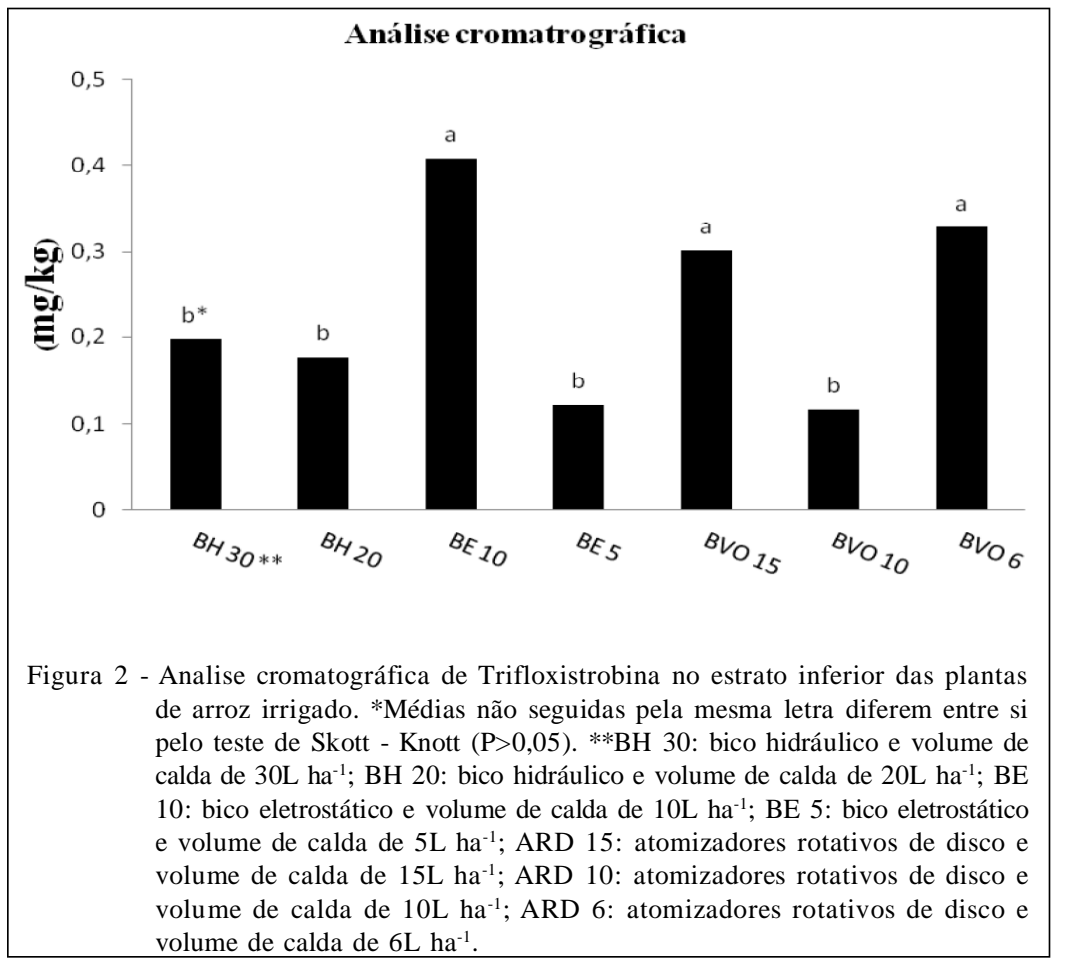

hidrossensíveis são limitados para avaliação de penetração para os bicos eletrostáticos e atomizadores rotativos de disco.

\section{REFERÊNCIAS}

ANASTASSIADES, M. et al. Fast and easy multiresidue method employing acetonitrile extraction/partitioning and "dispersive solid-phase extraction" for the determination of pesticide residues in produce. Journal of the Association of Official Analytical Chemists, v.86, p.412-431, 2003. Disponível em: <http://doi.editoracubo.com.br/10.4322/sc.2011.004>. Acesso em: 23 de jan. 2012.

ANTUNIASSI, U.R.; BAIO, F.H.R. Tecnologia de aplicação de defensivos. In: VARGAS, L.; ROMAN, E.S. Manual de manejo e controle de plantas daninhas. Passo Fundo: Embrapa Trigo, 2008. p.174-175.

BALAN, M.G. et al. Depósito e perdas de calda em sistema de pulverização com turboatomizador em videira. Engenharia Agrícola, v. 26, n. 2, p.470-477, 2006. Disponível em: <http:/ /www.scielo.br/scielo.php?script=sci_arttext\&pid=S0100$69162006000200015 \& \operatorname{lng}=\mathrm{en} \& \mathrm{nrm}=\mathrm{iso} \& \mathrm{t} \operatorname{lng}=\mathrm{pt}>$. Acesso em: 15 dez. 2011. doi: 10.1590/S0100-69162006000200015.

BALARDIN, R.S.; BORIN, R.C. Doenças na cultura do arroz irrigado. Santa Maria: UFSM, 2001. 48p.

CARVALHO, W.P.A. Estudo comparativo entre métodos de amostragem de gotas para determinação de faixa de deposição nas aplicações de produtos líquidos. 1995. 64f. Dissertação (Mestrado em Agronomia Energia na Agricultura) - Programa de Pós-graduação em Agronomia Energia na Agricultura, Universidade Estadual Paulista, SP.

COUNCE, P.A. et al. A uniform, objective, and adaptive system for expressing rice development. Crop Science, v.40, n.2, p.436-443, 2000. Disponível em: <http://cses.uark.edu/ 1815.htm>. Acesso em: 03 dez. 2011.

CONAB (Companhia Nacional de Abastecimento). Disponível em: <http://www.conab.gov.br/OlalaCMS/uploads/arquivos/ 12_06_12_16_15_32_boletim_portugues_junho_2012.pdf $>$. Online. Acesso em: 21 de jun. 2012.

CUNHA, J.P.A.R. et al. Deposição de calda pulverizada na cultura da soja promovida pela aplicação aérea e terrestre. Engenharia Agrícola, v.31, n.3, p.343-351, 2011. Disponível em: <http://www.scielo.br/ scielo.php?pid=S0100-69162011000200014\&script=sci_arttext $>$. Acesso em: 03 dez. 2011. doi: 10.1590/S0100-69162011000200014.

CUNHA, J.P.A.R.; RUAS, R.A.A. Uniformidade de distribuição volumétrica de pontas de pulverização de jato plano duplo com indução de ar. Pesquisa Agropecuária Tropical, v.36, p.6166, 2006. Disponível em: <http://www.revistas.ufg.br/index.php/ pat/article/view/2173/2121>. Acesso em: 19 out. 2011.

CUNHA, J.P.A.R. et al. Avaliação de estratégias para redução da deriva de agrotóxicos em pulverizações hidráulicas. Planta Daninha, v.21, n.2, p.325-332, 2003. Disponível em: <http:/ /www.scielo.br/pdf/pd/v21n2/a19v21n2.pdf >. Acesso em: 19 out. 2011. doi: 10.1590/S0100-83582003000200019. 
FAO (Organizações das Nações Unidas para Agricultura e Alimentação). Disponível em: <http://www.fao.org/docrep/011/ ai474e/ai474e05.htm\#34>. Acesso em: 21 jun. 2012.

MATUO, T. et al. Tecnologia de aplicação de defensivos agrícolas e equipamentos e técnicas de aplicação. In: ABEAS. Curso de proteção de plantas, Mod. 2, especialização por tutoria à distância. Brasília: ABEAS, 2001. p.71.
MENEGHETTI, R.C. Tecnologia de aplicação de fungicidas na cultura do trigo. 2006. 56f. Dissertação (Mestrado em Agronomia) - Programa de Pós-graduação em Agronomia, Universidade Federal de Santa Maria, RS.

OZEKI, Y. Manual de aplicação aérea. São Paulo: Edição do Autor, 2006. 101p. (Boletim Técnico). 